\title{
LINEAR COMBINATIONS OF PRIME POWERS IN BINARY RECURRENCE SEQUENCES
}

\author{
CS. BERTÓK, L. HAJDU, I. PINK, AND ZS. RÁBAI
}

\begin{abstract}
We give finiteness results concerning terms of linear recurrence sequences having a representation as linear combination, with fixed coefficients, of powers of fixed primes. On one hand, under certain conditions, we give effective bounds for the terms of binary recurrence sequences with such a representation. On the other hand, in case of some special binary recurrence sequences, all terms having a representation as sums of powers of 2,3 and $2,3,5$ are explicitly determined.
\end{abstract}

\section{INTRODUCTION}

Searching for specific terms of linear recurrence sequences has a long history and a rich literature. Here we give only a few examples, and refer the interested reader to the book [13] or the papers $[5,6,11]$ and the references given there.

Pethő [8] and Shorey and Stewart [12] independently proved that under certain natural assumptions, a linear recurrence sequence may contain only finitely many perfect powers. In case of some special, famous sequences all perfect powers have been determined. In case of the Pell sequence $P_{n}$, Pethö [9] proved that it does not contain non-trivial powers. Bugeaud, Mignotte and Siksek [3] proved that the Fibonacci-sequence $F_{n}$ contains only the powers $0,1,8,144$, and the only powers in the sequence of Lucas numbers $L_{n}$ are 1,4 . Pethő and Tichy [10] determined all Fibonacci numbers of the form $p^{a}+p^{b}+p^{c}$, where $p$ is a fixed prime. Kovács [5] found all combinatorial numbers of certain shapes among the terms of $F_{n}, L_{n}, P_{n}$ and $Q_{n}$ (the associated Pell-sequence).

Recently, Marques and Togbé [6] found all Fibonacci- and Lucas numbers of the form $2^{a}+3^{b}+5^{c}$ with $c \geq \max (a, b)$. Note that in their

2010 Mathematics Subject Classification. 11B37, 11D72.

Key words and phrases. recurrence sequences, sums of prime powers.

Research supported in part by the OTKA grants K100339 and K115479. The research was also granted by the Austrian science found (FWF) under the project P 24801-N26. 
approach, the assumption $c \geq \max (a, b)$ is unavoidable. Finally, we mention a result of Sanchez and Luca [11], who among others showed that the only Fibonacci number of the shape $\pm m ! \pm 2^{a} 3^{b} 5^{c} 7^{d}$ is $F_{24}=$ $8 !+2^{5} 3^{3} 5^{0} 7^{1}$. Sanchez and Luca noted that by their method they cannot solve the equation $F_{n}=2^{a}+3^{b}$.

The purpose of this paper is twofold. On one hand, under certain assumptions, we give general finiteness result for the solutions of the equation

$$
U_{n}=b_{1} p_{1}^{a_{1}}+\cdots+b_{s} p_{s}^{a_{s}}
$$

in non-negative integers $a_{1}, \ldots, a_{s}$, where $U_{n}$ is a binary recurrence sequence of positive discriminant, $b_{1}, \ldots, b_{s}$ are fixed integers, and $p_{1}, \ldots, p_{s}$ are given primes. Our result covers the case where some (possibly all) of the $p_{i}$-s are equal. On the other hand, we completely solve the above equation for the cases where $U_{n}$ is one of the sequences $F_{n}, L_{n}, P_{n}, Q_{n}$, and $\left(p_{1}, \ldots, p_{s}\right)$ is $(2,3)$ or $(2,3,5)$. In particular, this result extends the theorem of Marques and Togbé [6], and solves the above mentioned problem of Sanchez and Luca [11]. To prove our theorems, we apply Baker's method and involved local arguments.

\section{Notation AND MAIN RESUlts}

The sequence $U_{n}=U\left(A, B, U_{0}, U_{1}\right)$ is called a binary linear recurrence sequence if the relation

$$
U_{n}=A U_{n-1}+B U_{n-2}(n \geq 2)
$$

holds, where $A B \neq 0, U_{0}, U_{1}$ are fixed rational integers and $\left|U_{0}\right|+$ $\left|U_{1}\right|>0$. The polynomial $f(x)=x^{2}-A x-B$ is called the companion polynomial of the sequence $U_{n}$. Let $D=A^{2}+4 B$ be the discriminant of $f$. We call $D$ the discriminant of the sequence $U_{n}$. The roots of the companion polynomial are denoted by $\alpha$ and $\beta$. Throughout the paper, we shall always assume that $|\alpha| \geq|\beta|$. Using this notation, if $D \neq 0$ then as it is well known, we can write

$$
U_{n}=\frac{a \alpha^{n}-b \beta^{n}}{\alpha-\beta}
$$

for $n \geq 0$, where $a=U_{1}-U_{0} \beta$ and $b=U_{1}-U_{0} \alpha$. Note that $\alpha-\beta=\sqrt{D}$. The sequence $U_{n}$ is called non-degenerate, if $a b \alpha \beta \neq 0$ and $\alpha / \beta$ is not a root of unity.

Theorem 2.1. Let $U_{n}$ be a non-degenerate binary recurrence sequence with positive discriminant, $p_{1} \leq p_{2} \leq \cdots \leq p_{s}$ be given, not necessarily distinct prime numbers and $b_{1}, \ldots, b_{s}$ be nonzero integers. Put $T=$ 
$\max _{1 \leq i \leq s}\left|b_{i}\right|$. Using the above notation, assume further that $\log \left(\left|a / b_{s} \sqrt{D}\right|\right)$, $\log |\alpha|$ and $\log p_{s}$ are linearly independent over the rationals.

Consider the equation

$$
U_{n}=b_{1} p_{1}^{a_{1}}+b_{2} p_{2}^{a_{2}} \cdots+b_{s} p_{s}^{a_{s}}
$$

in non-negative integers $n, a_{1}, \ldots, a_{s}$. Let $0<\varepsilon<1$, and write $H_{\varepsilon}$ for the set of those solutions $\left(n, a_{1}, \ldots, a_{s}\right)$, for which $a_{s}=\max _{1 \leq i \leq s} a_{i}$, and $a_{i}<(1-\varepsilon) a_{s}$ for those $i=1, \ldots, s-1$ for which $p_{i}=p_{s}$.

Then $H_{\varepsilon}$ is finite, and for all $\left(n, a_{1}, \ldots, a_{s}\right)$ in $H_{\varepsilon}$ we have

$$
\max \left\{n, a_{1}, \ldots, a_{s}\right\}<C,
$$

where $C$ is an effectively computable constant depending only on $\varepsilon, A$, $B, U_{0}, U_{1}, T, s, p_{s}$.

Remark 1. Obviously, if $p_{s}$ is greater than all the other primes $p_{1}, \ldots, p_{s-1}$, then $H_{\varepsilon}$ is independent of $\varepsilon$.

Remark 2. The condition that $\log \left(\left|a / b_{s} \sqrt{D}\right|\right), \log |\alpha|$ and $\log p_{s}$ are linearly independent over the rationals is necessary. This is shown by the following example. Put $A=5, B=-6, U_{0}=0$ and $U_{1}=1$. Then $\alpha=3, \beta=2, a=b=\sqrt{D}=1$ and we have

$$
U_{n}=3^{n}-2^{n} \quad(n \geq 0) .
$$

Take $s=2, p_{1}=2, p_{2}=3, b_{1}=-1$ and $b_{2}=1$. Then the equation

$$
U_{n}=b_{1} p_{1}^{a_{1}}+b_{2} p_{2}^{a_{2}}
$$

has infinitely many solutions given by $\left(n, a_{1}, a_{2}\right)=(n, n, n)(n \geq 0)$, which belong to $H_{\varepsilon}$ for any $\varepsilon$. Observe that now $\log \left(\left|a / b_{s} \sqrt{D}\right|\right), \log |\alpha|$ and $\log p_{s}$ are linearly dependent over $\mathbb{Q}$, however, all the other conditions of Theorem 2.1 are satisfied.

The sequences corresponding to the particular choices

$$
\left(A, B, U_{0}, U_{1}\right)=(1,1,0,1),(1,1,2,1),(2,1,0,1),(2,1,1,1)
$$

are the Fibonacci-sequence $F_{n}$, the sequence of Lucas-numbers $L_{n}$, the Pell-sequence $P_{n}$ and the associated Pell sequence $Q_{n}$, respectively. Our next theorem extends the above mentioned result of Marques and Togbé [6]: we get rid of the condition $c \geq \max (a, b)$ in the equation

$$
U_{n}=2^{a}+3^{b}+5^{c}
$$

with $U_{n}=F_{n}, L_{n}$, and we consider the cases $U_{n}=P_{n}, Q_{n}$, as well. Further, we completely solve the above equation (for these four sequences) when the right hand side is replaced by $2^{a}+3^{b}$. In particular, 
if $U_{n}=F_{n}$, our result solves the previously mentioned problem of Sanchez and Luca [11].

Note that the solutions of the equation $U_{n}=2^{a}$ are also known for these sequences: for $U_{n}=F_{n}, L_{n}$ they can be obtained from the already mentioned results of Bugeaud, Mignotte and Siksek [3] (the solutions are $(n, a)=(1,0),(2,0),(5,3)$ and $(n, a)=(0,2),(1,0)$, respectively), for $U_{n}=P_{n}$ the only solution $(n, a)=(1,0)$ is given by the result of Pethő [9], while for $U_{n}=Q_{n}$ the only solutions are $(n, a)=(0,0)$ and $(1,0)$, since all the terms of $Q_{n}$ with $n>0$ are odd and greater than one.

Theorem 2.2. Let $U_{n}$ be one of $F_{n}, L_{n}, P_{n}, Q_{n}$. Then the solutions of the equation

$$
U_{n}=2^{a}+3^{b}
$$

in non-negative integers $n, a, b$ are given by Table 1 .

\begin{tabular}{|c|c|}
\hline & $(n, a, b)$ \\
\hline$F_{n}$ & $(3,0,0),(4,1,0),(5,1,1),(5,2,0),(7,2,2),(11,3,4)$ \\
\hline$L_{n}$ & $(0,0,0),(3,0,1),(2,1,0),(5,1,2)$, \\
& $(7,1,3),(4,2,1),(13,9,2),(5,3,1)$ \\
\hline$P_{n}$ & $(2,0,0),(3,1,1),(5,1,3),(3,2,0),(9,8,6)$ \\
\hline$Q_{n}$ & $(2,1,0),(3,2,1),(4,3,2),(4,4,0),(5,5,2)$ \\
\hline
\end{tabular}

TABLE 1. Solutions of equation (4)

Further, still with $U_{n}$ being one of $F_{n}, L_{n}, P_{n}, Q_{n}$, the solutions of the equation

$$
U_{n}=2^{a}+3^{b}+5^{c}
$$

in non-negative integers $n, a, b, c$ are those occurring in Table 2.

\begin{tabular}{|c|c|}
\hline & $(n, a, b, c)$ \\
\hline$F_{n}$ & $(4,0,0,0),(5,0,1,0),(6,1,0,1),(9,1,3,1)$, \\
& $(6,2,1,0),(9,3,0,2),(12,4,1,3),(9,5,0,0)$ \\
\hline$L_{n}$ & $(2,0,0,0),(4,0,0,1),(7,0,1,2),(5,0,2,0),(7,0,3,0)$, \\
& $(3,1,0,0),(6,2,2,1),(6,3,2,0),(6,4,0,0)$ \\
\hline$P_{n}$ & $(3,0,1,0),(5,0,3,0),(4,1,2,0),(5,0,1,2),(4,2,1,1)$, \\
& $(4,3,1,0),(6,6,0,1),(8,8,3,3),(10,10,6,4)$ \\
\hline$Q_{n}$ & $(2,0,0,0),(3,0,0,1)$ \\
\hline
\end{tabular}

TABLE 2. Solutions of equation (5) 


\section{Proofs}

To prove Theorem 2.1, we shall use Baker's method. First we need to introduce some notation.

For an algebraic number $\eta$ of degree $d$ over $\mathbb{Q}$, we define as usual the absolute logarithmic height of $\eta$ by the formula

$$
h(\eta)=\frac{1}{d}\left(\log \left|a_{0}\right|+\sum_{i=1}^{d} \log \max \left(1,\left|\eta^{(i)}\right|\right)\right),
$$

where $a_{0}$ is the leading coefficient of the minimal polynomial of $\eta$ over $\mathbb{Z}$ and the $\eta^{(i)}$-s are the conjugates of $\eta$ in the field of complex numbers. In particular, if $\eta=p / q$ is a rational number with $\operatorname{gcd}(p, q)=1$, then $h(\eta)=\log \max \{|p|,|q|\}$.

Now we state a Baker-type result of Matveev [7], which will be used in the proof of our Theorem 2.1.

Lemma 3.1 (Matveev). Assume that $\gamma_{1}, \ldots, \gamma_{t}$ are nonzero algebraic numbers in a real algebraic number field $\mathbb{K}$ of degree $d_{\mathbb{K}}, d_{1}, \ldots, d_{t}$ are rational integers, and

$$
\Lambda:=\gamma_{1}^{d_{1}} \ldots \gamma_{t}^{d_{t}}-1
$$

is not zero. Set

$$
B \geq \max \left\{\left|d_{1}\right|, \ldots,\left|d_{t}\right|\right\}
$$

and

$$
A_{i} \geq \max \left\{d_{\mathbb{K}} h\left(\gamma_{i}\right),\left|\log \gamma_{i}\right|, 0.16\right\}, \text { for all } i=1, \ldots, t .
$$

Then we have

$$
|\Lambda|>\exp \left(-1.4 \cdot 30^{t+3}(t+1)^{4.5} d_{\mathbb{K}}^{2} A_{1} \ldots A_{t}\left(1+\log d_{\mathbb{K}}\right)(1+\log B)\right) .
$$

Proof of Theorem 2.1. First, for later use observe that our assumptions imply that

(6)

$$
\left|b_{1} p_{1}^{a_{1}}+\cdots+b_{s-1} p_{s-1}^{a_{s-1}}\right|=p_{s}^{a_{s}}\left|b_{1} \frac{p_{1}^{a_{1}}}{p_{s}^{a_{s}}}+\cdots+b_{s-1} \frac{p_{s-1}^{a_{s-1}}}{p_{s}^{a_{s}}}\right| \leq(s-1) T p_{s}^{\left(1-\delta_{1}\right) a_{s}}
$$

where

$$
\delta_{1}=\min \left(\varepsilon, 1-\max _{p_{i}<p_{s}}\left(\log \left(p_{i}\right) / \log \left(p_{s}\right)\right)\right) .
$$

If $p_{1}=\cdots=p_{s}$ then we take $\delta_{1}=\varepsilon$. Since $D>0$ and $U_{n}$ is assumed to be non-degenerate, we have

$$
|\alpha|>|\beta|
$$


Note that by our assumptions we also have $|\alpha|>1$. A simple calculation shows that if

$$
\left|1-\frac{b \beta^{n}}{a \alpha^{n}}\right| \leq \frac{1}{2}
$$

then $n$ is bounded by a constant depending only on $A, B, U_{0}, U_{1}$. Then since by (6) we get that the right hand side of (3) tends to infinity as $a_{s}$ tends to infinity, we obtain that $a_{s}$ is bounded by a constant depending only on $A, B, U_{0}, U_{1}, s, p_{s}$. Hence the statement follows in this case. So we may assume that

$$
\left|1-\frac{b \beta^{n}}{a \alpha^{n}}\right|>\frac{1}{2}
$$

Then (3) implies that

$$
\left|\frac{a \alpha^{n}}{2 \sqrt{D}}\right|<\left|\frac{a \alpha^{n}}{\sqrt{D}}\right|\left|1-\frac{b \beta^{n}}{a \alpha^{n}}\right|=\left|U_{n}\right| \leq s T p_{s}^{a_{s}} .
$$

This gives

$$
n \leq \frac{\log \frac{2 \sqrt{D} s T}{|a|}+a_{s} \log p_{s}}{\log |\alpha|}
$$

If

$$
\log \frac{2 \sqrt{D} s T}{|a|}>a_{s} \log p_{s}
$$

then our theorem trivially follows. So we may assume the contrary, when by (9) we obtain

$$
n \leq \frac{2 a_{s} \log p_{s}}{\log |\alpha|}
$$

If $|\beta|>1$ we need more. In this case, if

$$
\log \frac{2 \sqrt{D} s T}{|a|}>\frac{a_{s} \log p_{s}(\log |\alpha|-\log |\beta|)}{2 \log |\beta|}
$$

then the theorem easily follows. So we may assume the opposite, when by (9) we get

$$
n \leq \frac{a_{s} \log p_{s}(\log |\alpha|+\log |\beta|)}{2 \log |\alpha| \log |\beta|} .
$$

Now we rewrite (3) as

$$
\frac{a}{b_{s} \sqrt{D}} \alpha^{n} p_{s}^{-a_{s}}-1=\frac{b_{1} p_{1}^{a_{1}}+\cdots+b_{s-1} p_{s-1}^{a_{s-1}}+\frac{b \beta^{n}}{\sqrt{D}}}{b_{s} p_{s}^{a_{s}}} .
$$


In case of $|\beta| \leq 1$ we obviously have

$$
\frac{|\beta|^{n}}{p_{s}^{a_{s}}} \leq p_{s}^{-a_{s}}
$$

If $|\beta|>1$, then (11) implies

$$
\frac{|\beta|^{n}}{p_{s}^{a_{s}}} \leq p_{s}^{-\delta_{2} a_{s}}
$$

where

$$
\delta_{2}=1-\frac{\log |\alpha|+\log |\beta|}{2 \log |\alpha|} .
$$

Note that $1>\delta_{2}>0$. Using the above inequalities together with (6), from (12) we get

$$
|\Lambda| \leq c_{2} p_{s}^{-\delta a_{s}}
$$

where $\Lambda=\frac{a}{b_{s} \sqrt{D}} \alpha^{n} p_{s}^{-a_{s}}-1, \delta=\min \left\{\delta_{1}, \delta_{2}\right\}$ and $c_{2}=(s-1) T+|b| / \sqrt{D}$.

On the other hand, since by our assumption

$$
\log \frac{|a|}{\left|b_{s}\right| \sqrt{D}}, \quad \log |\alpha|, \quad \log p_{s}
$$

are linearly independent over the rationals, we have $\Lambda \neq 0$. Hence, in view of (10) Lemma 3.1 gives that

$$
|\Lambda|>\exp \left(-c_{3} \log a_{s}\right)
$$

holds with some constant $c_{3}$ depending only on $A, B, U_{0}, U_{1}$ and $p_{s}$.

Combining the upper and lower estimates for $|\Lambda|$, we get an upper bound for $a_{s}$ in terms of $\varepsilon, A, B, U_{0}, U_{1}, T, s$ and $p_{s}$. Hence in view of (10) the statement clearly follows.

Proof of Theorem 2.2. Since we apply a similar method (local arguments) for every equation, we only demonstrate our approach for the case of $F_{n}=2^{a}+3^{b}$. For the remaining equations we only list the moduli used.

We rewrite the equation $F_{n}=2^{a}+3^{b}$ as

$$
\alpha^{n}-\beta^{n}-\sqrt{5} \cdot 2^{a}-\sqrt{5} \cdot 3^{b}=0,
$$

where $\alpha=\frac{1+\sqrt{5}}{2}$ and $\beta=\frac{1-\sqrt{5}}{2}$. First we list all "small" solutions of this equation, i.e. the solutions with $n, a, b \leq 100$. In fact, we just find the solutions given in Table 1 . We observe that in every "small" solution the exponent of 2 is at most 3, and instead of (13) we consider the equation

$$
\alpha^{n}-\beta^{n}-\sqrt{5} \cdot 2^{4} \cdot 2^{a_{1}}-\sqrt{5} \cdot 3^{b}=0 .
$$


We suspect that equation (14) has no solutions in non-negative integers $n, a_{1}, b$. To show this, we work in the ring of integers $O_{K}$ of the number field $K=\mathbb{Q}(\sqrt{5})$. We exhibit a modulus $m \in O_{K}$ such that (14) has no solutions already modulo $m$. (Here, and elsewhere from this point on, all congruences are to be taken in $O_{K}$.) As we shall see,

$$
m=2^{4} \cdot 5 \cdot 7 \cdot 13 \cdot 17 \cdot 19 \cdot 29 \cdot 31 \cdot 37 \cdot 43 \cdot 73
$$

is an appropriate choice. For this, consider equation (14) modulo $m_{1}=$ $2^{4}$. One can easily check that $\operatorname{ord}_{2^{4}}(\alpha)=\operatorname{ord}_{2^{4}}(\beta)=24, \operatorname{ord}_{2^{4}}(3)=4$, where $\operatorname{ord}_{q}(\gamma)$ is the smallest non-negative integer $j$ for which $\gamma^{j} \equiv 1$ $(\bmod q)$ for $q, \gamma \in O_{K}$. (In all cases we consider, such a $j$ will always exist.) Since, $\sqrt{5} \cdot 2^{4} \cdot 2^{a_{1}} \equiv 0\left(\bmod 2^{4}\right)$, we only need to consider $24 \cdot 4=96$ possibilities. By checking all possible cases, we get that there are only eight solutions modulo $2^{4}$. These solutions are given by

$$
\begin{gathered}
(n, b) \equiv(1,0),(2,0),(4,1),(11,2),(13,2), \\
(14,2),(16,3),(23,0) \quad(\bmod (24,4)),
\end{gathered}
$$

where here and later on by

$$
\left(u_{1}, \ldots, u_{k}\right) \equiv\left(v_{1}, \ldots, v_{k}\right) \quad\left(\bmod \left(q_{1}, \ldots, q_{k}\right)\right)
$$

we mean that $u_{i} \equiv v_{i}\left(\bmod q_{i}\right)$ for every $i=1, \ldots, k$. We consider only the case $n \equiv 4(\bmod 24)$ and $b \equiv 1(\bmod 4)$, the other cases can be handled similarly. Now putting $n=24 n_{1}+4$ and $b=4 b_{1}+1$, from (14) we get

$$
\alpha^{4} \cdot\left(\alpha^{24}\right)^{n_{1}}-\beta^{4} \cdot\left(\beta^{24}\right)^{n_{1}}-\sqrt{5} \cdot 2^{4} \cdot 2^{a_{1}}-\sqrt{5} \cdot 3 \cdot\left(3^{4}\right)^{b_{1}}=0 .
$$

Take $m_{2}=5$. As we have $\operatorname{ord}_{5}\left(\alpha^{24}\right)=\operatorname{ord}_{5}\left(\beta^{24}\right)=5, \operatorname{ord}_{5}(2)=4$ and $\operatorname{ord}_{5}\left(3^{4}\right)=1$, we need to consider $5 \cdot 4=20$ cases. By a simple computation we get that

$$
\left(n_{1}, a_{1}\right) \equiv(1,3),(2,0),(3,2),(4,1) \quad(\bmod (5,4)) .
$$

We pick up the case $n_{1} \equiv 3(\bmod 5)$ and $a_{1} \equiv 2(\bmod 4)$, the other cases can be treated similarly. Now letting $n_{1}=5 n_{2}+3$ and $a_{1}=$ $4 a_{2}+2,(15)$ yields

$$
\alpha^{76} \cdot\left(\alpha^{120}\right)^{n_{2}}-\beta^{76} \cdot\left(\beta^{120}\right)^{n_{2}}-\sqrt{5} \cdot 2^{6} \cdot\left(2^{4}\right)^{a_{2}}-\sqrt{5} \cdot 3 \cdot\left(3^{4}\right)^{b_{1}}=0 .
$$

We take $m_{3}=7$. As one can easily check, we have $\operatorname{ord}_{7}\left(\alpha^{120}\right)=$ $\operatorname{ord}_{7}\left(\beta^{120}\right)=2$ and $\operatorname{ord}_{7}\left(2^{4}\right)=\operatorname{ord}_{7}\left(3^{4}\right)=3$. That is, we have to consider $2 \cdot 3 \cdot 3=18$ cases. By doing this we get two solutions, namely

$$
\left(n_{2}, a_{2}, b_{1}\right) \equiv(0,0,0),(1,2,2) \quad(\bmod (2,3,3))
$$


Taking the first triplet (the other one can be handled similarly), putting $n_{2}=2 n_{3}, a_{2}=3 a_{3}$ and $b_{1}=3 b_{2}$, we obtain

$$
\alpha^{76} \cdot\left(\alpha^{240}\right)^{n_{3}}-\beta^{76} \cdot\left(\beta^{240}\right)^{n_{3}}-\sqrt{5} \cdot 2^{6} \cdot\left(2^{12}\right)^{a_{3}}-\sqrt{5} \cdot 3 \cdot\left(3^{12}\right)^{b_{2}}=0 .
$$

Now choose $m_{4}=13$. Then $\operatorname{ord}_{13}\left(\alpha^{240}\right)=\operatorname{ord}_{13}\left(\beta^{240}\right)=7$, and $\operatorname{ord}_{13}\left(2^{12}\right)=\operatorname{ord}_{13}\left(3^{12}\right)=1$. If we check all seven possibilities modulo $m_{4}=13$, we get that there are no solutions.

By following the same argument as above for the occurring possibilities, using the factors of $m$, we conclude that (14) has no solutions modulo $m$. This implies that in (13) we must have $a \leq 3$. From this point one can either use the same method again to solve the four new equations (with $a=0,1,2,3$ ), only in one free variable, or one can use elliptic curves for the same purpose. We follow the latter approach which, as we shall see, always becomes available whenever the number of free variables is reduced to one. Before coming to that point, we give some explanation about finding appropriate moduli to make the local argument work.

In general, to apply the local method to solve the arising exponential equations, we need to find a modulus $m$ such that the orders of $\alpha, \beta$, 2,3 and 5 with respect to $m$ are "small". To achieve such a modulus, we used the method described in [1] (based on the work of Erdös, Pomerance and Schmutz [4] on the "small" values of Carmichael's $\lambda$ function). Typically, if $m=p_{1}^{\alpha_{1}} \cdot \ldots \cdot p_{l}^{\alpha_{l}}$ with rational primes $p_{1}, \ldots, p_{l}$, then if $p_{k}-1$ has only "small" prime divisors for every $1 \leq k \leq l$, then the condition above is satisfied. For details (for the rational case) see [1].

In Table 3 we give the moduli used for our equations. Further, we also indicate what is the conclusion we can draw by the help of that modulus. In the table we use the notation

$$
\begin{gathered}
M_{1}=7 \cdot 13 \cdot 17 \cdot 19 \cdot 29 \cdot 31 \cdot 37 \cdot 73, \\
M_{2}=M \cdot 3^{7} \cdot 5 \cdot 11 \cdot 41 \cdot 12289 \cdot 17497 \cdot 18433 \cdot 65537, \\
M_{3}=M \cdot 2^{11} \cdot 3^{7} \cdot 12289 \cdot 17497 \cdot 18433, \\
M_{4}=M \cdot 2 \cdot 3^{7} \cdot 11 \cdot 41 \cdot 12289 \cdot 17497, \\
M_{5}=M \cdot 2^{12} \cdot 3 \cdot 11 \cdot 41 \cdot 39367 \cdot 65537,
\end{gathered}
$$

where

$M=7 \cdot 13 \cdot 17 \cdot 19 \cdot 29 \cdot 31 \cdot 37 \cdot 43 \cdot 73 \cdot 97 \cdot 109 \cdot 163 \cdot 193 \cdot 257 \cdot 433 \cdot 487 \cdot 577$.

$.769 \cdot 1153 \cdot 1297 \cdot 1459 \cdot 2593 \cdot 2917 \cdot 3137 \cdot 3457 \cdot 3889 \cdot 10369$. 


\begin{tabular}{|c|c|c|c|c|c|}
\hline seq. & representation & modulus & conclusion & modulus & conclusion \\
\hline \multirow[b]{2}{*}{$F_{n}$} & $2^{a}+3^{b}$ & $M_{1} \cdot 2^{4} \cdot 5 \cdot 43$ & $a \leq 3$ & - & - \\
\hline & $2^{a}+3^{b}+5^{c}$ & $\begin{array}{c}M_{1} \cdot 2^{6} \cdot 97 \cdot \\
\cdot 109 \cdot 193 \cdot 769\end{array}$ & $a \leq 5$ & $\begin{array}{c}M_{1} \cdot 2 \cdot 3^{4} \cdot \\
.97 \cdot 109 \cdot 769\end{array}$ & $b \leq 3$ \\
\hline \multirow[t]{2}{*}{$L_{n}$} & $2^{a}+3^{b}$ & $\begin{array}{c}M_{1} \cdot 3^{4} \cdot 5 \cdot 43 \cdot \\
\cdot 97 \cdot 109 \cdot 163 \cdot \\
\cdot 193 \cdot 257 \cdot 433 \cdot \\
\cdot 487 \cdot 577 \cdot 769 \cdot 3889\end{array}$ & $b \leq 3$ & - & - \\
\hline & $2^{a}+3^{b}+5^{c}$ & $\begin{array}{c}M_{1} \cdot 2^{5} \cdot \\
\cdot 97 \cdot 109 \cdot 193\end{array}$ & $a \leq 4$ & $\begin{array}{c}M_{1} \cdot 2^{5} \cdot 3^{4} \cdot 11^{2} \cdot \\
\cdot 41 \cdot 61 \cdot 67 \\
\cdot 71 \cdot 109 \cdot 271\end{array}$ & $b \leq 3$ \\
\hline \multirow[b]{2}{*}{$P_{n}$} & $2^{a}+3^{b}$ & $M_{2}$ & $b \leq 6$ & - & - \\
\hline & $2^{a}+3^{b}+5^{c}$ & $M_{3}$ & $\begin{array}{c}a \leq 10 \text { or } \\
b \leq 6\end{array}$ & $\begin{array}{c}M_{4} \text { resp. } \\
M_{5}\end{array}$ & $\begin{array}{c}b \leq 6 \text { resp. } \\
a \leq 11\end{array}$ \\
\hline \multirow[t]{2}{*}{$Q_{n}$} & $2^{a}+3^{b}$ & $\begin{array}{l}3^{3} \cdot 5 \cdot 7 \cdot 13 \cdot \\
\cdot 97 \cdot 109 \cdot 193\end{array}$ & $b \leq 2$ & - & - \\
\hline & $2^{a}+3^{b}+5^{c}$ & $2^{2}$ & $a \leq 1$ & $\begin{array}{c}3 \cdot 7 \cdot 11 \cdot 13 \cdot 17 \cdot \\
\cdot 19 \cdot 31 \cdot 37 \cdot 73\end{array}$ & $b=0$ \\
\hline
\end{tabular}

TABLE 3 . Used moduli and the conclusion drawn for the equations considered

As we have mentioned already, it is sufficient to reduce the number of free variables on the right side of equations (4) and (5) to one by the local argument. Then by the help of the identities

$$
L_{n}^{2}-5 F_{n}^{2}= \pm 4 \quad \text { and } \quad 2 P_{n}^{2}-Q_{n}^{2}= \pm 1
$$

one can reduce the problem to finding integral points on elliptic curves. We shall demonstrate our method by exhibiting a particular case. Recall that in case of the equation $F_{n}=2^{a}+3^{b}$ our local arguments implied $a=0,1,2,3$. As an example, we take $a=3$, the other cases can be handled similarly. In this case we need to solve the equation

$$
F_{n}=3^{b}+8
$$

We can write $b=2 k$ or $b=2 k+1$ with some integer $k$. Put $y=L_{n}$ and $x=3^{k}$. Then from the first identity in (18) in case of $b=2 k$ we get

$$
y^{2}=5 x^{4}+80 x^{2}+320 \pm 4,
$$

while for $b=2 k+1$ we obtain

$$
y^{2}=45 x^{4}+240 x^{2}+320 \pm 4 .
$$


Using the Magma [2] procedure IntegralQuarticPoints, we get the integer solutions $x, y$ of these equations. Namely, if $(x, y)$ is a solution to any of the above equations in positive integers, then it is one of

$$
(x, y)=(0,18),(9,199),(15,521) .
$$

Among these solutions we select those where $x$ is a non-negative power of 3 . In this case the only such solution is $(x, y)=(9,199)$. We conclude that equation (19) has the only solution $b=4$ (with $n=11$ ).

In case of all the other equations (4) and (5), after reducing the number of variables on the right hand sides to one, the above method worked and provided the solutions in Tables 1 and 2 .

\section{REFERENCES}

[1] Cs. Bertók and L. Hajdu, A Hasse-type principle for exponential Diophantine equations and its applications, Math. Comp., electronically published on July 16, 2014, DOI: http://dx.doi.org/10.1090/S0025-5718-2014-02815-9 (to appear in print).

[2] W. Bosma, J. Cannon, and C. Playoust, The Magma algebra system. I. The user language, J. Symbolic Comput. 24 (1997), 235-265.

[3] Y. Bugeaud, M. Mignotte, S. Siksek Classical and modular approaches to exponential Diophantine equations. I. Fibonacci and Lucas perfect powers, Ann. Math. 163 (2006), 969-1018.

[4] P. Erdős, C. Pomerance, E. Schmutz, Carmichael's lambda function, Acta Arithmetica 58.4 (1991), 363-385.

[5] T Kovács, Combinatorial numbers in binary recurrences, Period. Math. Hungar. 58 (2009), 83-98.

[6] D. Marques and A. Togbé, Fibonacci and Lucas numbers of the form $2^{a}+3^{b}+5^{c}$, Proc. Japan Acad. Ser. A 89 (2013), 47-50.

[7] E. M. Matveev, An explicit lower bound for a homogeneous rational linear form in the logarithms of algebraic numbers II, Izv. Ross. Akad. Nauk Ser. Mat. 64 (2000), 125-180; translation in Izv. Math. 64 (2000), 1217-1269.

[8] A. Pethö, Perfect powers in second order linear recurrences, J. Number Theory 15 (1982), 5-13.

[9] A. Pethő, The Pell sequence contains only trivial perfect powers, Proc. Sets Graphs and Numbers. Coll. Math. Soc. Jnos Bolyai Vol. 60, 1992, pp. 561568.

[10] A. Pethö and R. F. Tichy, S-unit equations, linear recurrences and digit expansions, Publ. Math. Debrecen 42/1-2 (1993), 145-154.

[11] S. G. Sanchez and F. Luca, Linear combinations of factorials and S-units in a binary recurrence sequence, Ann. Math. Québec 38 (2014), 169-188.

[12] T. N. Shorey and C. L. Stewart, On the Diophantine equation $a x^{2 t}+b x^{t} y+$ $c y^{2}=d$ and pure powers in recurrence sequences, Math. Scand. 52 (1983), 24-36.

[13] T. N. Shorey, R. Tijdeman, Exponential Diophantine Equations, Cambridge University Press, Cambridge, 1986. 
Cs. Bertók, L. Hajdu

University of Debrecen, Institute of Mathematics

H-4010 Debrecen, P.O. Box 12.

HUNGARY

I. PINK

Institute of Mathematics, University of Debrecen H-4010 Debrecen, P.O. Box 12, Hungary

AND

University OF SALZBURG

HELLBRUNNERSTRASSE 34/I

A-5020 Salzburg, Austria

Zs. RÁBAI

University of Debrecen, Institute of Mathematics

H-4010 Debrecen, P.O. Box 12.

HUNGARY

E-mail address: bertok.csanad@science.unideb.hu

E-mail address: hajdul@science.unideb.hu

E-mail address: pinki@science.unideb.hu; istvan.pink@sbg.ac.at

E-mail address: zsrabai@science.unideb.hu 